\title{
Longitudinal changes in recalled perceived life threat after a natural disaster ${ }^{\dagger}$
}

Trond Heir, Auran Piatigorsky and Lars Weisæth

\section{Background}

Post-traumatic stress disorder (PTSD) diagnosis often depends on a retrospective, self-report of exposure to a life-threatening event.

\section{Aims}

To examine the stability of recalled perceived life threat in a community sample exposed to a distinct stressful event.

\section{Method}

Five hundred and thirty-two Norwegian citizens who experienced the 2004 South-East Asia tsunami completed a self-report questionnaire 6 and 24 months post-disaster. The questionnaire measured perceived life-threat intensity, exposure, immediate stress response, psychopathology, personality dimensions, self-efficacy and social support.

\section{Results}

Recalled threat intensity increased from 6 to 24 months $(P<0.001)$. Recall amplification was associated with lack of PTSD symptom improvement $(P<0.05)$, but not with degree of exposure, immediate stress response, mood or stress symptoms, personality, self-efficacy or social support.

\section{Conclusions}

Recall amplification of perceived life threat from a single stressful event occurs in the general population, it may hinder PTSD symptom improvement and it questions the diagnostic validity of PTSD.

\section{Declaration of interest}

None.
To receive a diagnosis of post-traumatic stress disorder (PTSD), both within the ICD-10 $10^{1}$ and the DSM-IV ${ }^{2}$ a person must have experienced a horrific and/or life-threatening event. Often a stressful event is self-reported to a mental health professional months or even years after the event has occurred. The accuracy of recalled threat is therefore essential for correct diagnosis. Until recently, stressful event memories have been considered stable and accurate, ${ }^{3}$ but research with military veterans question whether such memories are indelible. ${ }^{4-8}$ It is unclear whether war veteran studies are relevant to the general civilian population. ${ }^{9}$ Indeed, studies with civilian samples have inconsistent findings. ${ }^{10-12}$ This longitudinal recall research generally focuses on reported frequencies of experiencing several stressful events rather than reported intensities of experiencing a single stressful event. To our knowledge, no studies exist that address recalled life-threat stability in a community sample that experienced a distinct stressful event. Our sample of Norwegian tourists on Christmas holiday who were repatriated from South-East Asia shortly after the 2004 tsunami offers a unique opportunity to study the effects of a well-defined, sudden onset, short duration event. In this study, we aimed to examine the stability of recalled threat intensity from 6 months to 2 years post-disaster.

\section{Method}

\section{Study design}

A longitudinal study design was used. All adult Norwegian nationals who were at a disaster stricken area of Thailand during the 2004 South-East Asia tsunami were requested by mail to complete a questionnaire at 6 and 24 months post-disaster. The questionnaire queried domains pertinent to exposure, background variables and psychological functioning. ${ }^{13}$

\footnotetext{
†See editorial, pp. 479-480, this issue.
}

\section{Study population}

The study population consisted of Norwegian nationals over the age of 18 years who had resided in one of the following Thai areas during the tsunami: Khao Lak, Phi-Phi Island, Krabi Province and Phuket. These areas included the vast majority of exposed Norwegian tourists. Of the 84 Norwegians who died during the tsunami, 82 perished in these areas. The Norwegian police, mainly upon victims' arrivals at Norwegian airports, registered lists of names, personal identification numbers and places of residence during the disaster. After permission from the Norwegian Data Inspectorate and The Regional Committee for Medical Research Ethics, this information was made available for our study. Of 1511 people invited to participate in our study, $643(42.6 \%)$ returned a questionnaire at 6 months, $816(54.0 \%)$ responded at 24 months, and $532(35.2 \%)$ responded at both assessments. The response rate was much higher in subgroups that had resided in the most severely affected areas, and it was correspondingly lower among those who had been in locations that were less severely affected. ${ }^{13}$ We investigated lack of participation with follow-up telephone calls in a random sample of non-responders $(n=171)$. The most frequently reported reasons for not participating in the study were lack of interest or time (39.2\%) and not being directly affected by the disaster $(32.2 \%)$.

Of the individuals who responded at both assessments, 116 were excluded because of missing data on outcome variables. The remaining 416 participants were of similar age with a higher proportion of women compared with the 1095 individuals who were not included (Table 1). Also, with regard to education level, employment, family constellations, and pre-disaster contact with health professionals, the 416 participants did not significantly differ from the 511 individuals who were excluded from the study but responded at the first or second assessment. In the included sample, $58 \%$ had more than 12 years of education, $74 \%$ were employed and $68 \%$ were married or cohabiting. Also, $68 \%$ were travelling with their spouse or live-in partner, 39\% with their children who were under 18 years old and $12 \%$ with their parents. The reported prior tsunami lifetime prevalence of contact with 
Table 1 Characteristics of the total population, individuals included and not included, of Norwegian nationals who had resided in the actual disaster affected areas

\begin{tabular}{|c|c|c|c|}
\hline & $\begin{array}{c}\text { Total disaster population, } \\
\qquad n=1511\end{array}$ & $\begin{array}{l}\text { Participants in analyses, } \\
\qquad n=416\end{array}$ & $\begin{array}{l}\text { Individuals not in analyses, } \\
\qquad n=1095\end{array}$ \\
\hline Age, years: mean (range) & $44.8(18-82)$ & $44.2(18-82)$ & $45.0(18-81)$ \\
\hline \multicolumn{4}{|l|}{ Gender, $n(\%)$} \\
\hline Women & 714 (47) & $224(54)$ & $490(45)^{\mathrm{a}}$ \\
\hline Men & 797 (53) & $192(46)$ & $605(55)$ \\
\hline
\end{tabular}

a general practitioner, psychologist or psychiatrist for mental health concerns was $22 \%$. The participants were similar to the age- and gender-adjusted Norwegian population with regard to employment and marital status (www.ssb.no/english/). Participants had a greater than average education level and family constellation with children (www.ssb.no/english/).

\section{Measures}

\section{Recalled perceived life threat}

In both assessments, recalled threat intensity was measured by the question: 'How great do you think the danger was that you would die?' The participants responded on a five-point scale: 1 , none; 2 , small; 3 , moderate; 4 , great; 5 , overwhelming. This item correlated highly with other items that measured danger such as whether participants were caught, touched or chased by waves (Spearman $r=0.67)$ or had physical injuries inflicted $(r=0.56)$. Both of these correlations are above 0.5 , which is a commonly used cut-off for acceptable scale construct validity. ${ }^{14}$

\section{Exposure and immediate response to the disaster}

At the 6-month assessment, the questionnaire included a broad spectrum of tsunami experiences: whether a participant was caught, touched or chased by waves; had physical injuries inflicted; witnessed death and suffering of others; was uncertain of the fate of family members or close friends; and had a close relative or friend die. ${ }^{13}$ The questionnaire also included a 19 -item list that retrospectively surveyed the immediate responses to the tsunami. Participants were asked whether their immediate responses, among other emotional reactions, were characterised by fear, helplessness or horror. Each of the items was measured on a five-point scale: 0 , not at all; 1 , little; 2 , moderate; 3 , intense; 4 , extreme. A score of 3 or 4 on a fear, helplessness or horror item was considered as a positive response to Criterion A2 for PTSD in DSM-IV

\section{Impact of Event Scale-Revised}

In both assessments, the 22-item Impact of Event Scale-Revised $(\text { IES-R })^{15}$ was included to examine the presence and intensity of post-traumatic stress symptoms during the previous week. The participants responded to each item on a five-point Likert scale regarding their experience with the tsunami. The IES-R total and subscale (intrusion, avoidance, hyperarousal) symptom scores were used as semi-continuous measures of symptom severity. We excluded a measure from analyses when a participant responded with $25 \%$ missing data. In a post hoc evaluation of the clinical importance of an IES-R mean value change, we examined changes in the proportion of participants with IES-R score $\geqslant 33$, which is a cut-off recommended to provide the best diagnostic accuracy. ${ }^{16}$

\section{General Health Questionnaire}

The 28-item General Health Questionnaire (GHQ-28) ${ }^{17}$ was used as a measure of general psychopathology at both assessments. We used GHQ-28 total and subscale (somatisation, anxiety, social dysfunction, depression) scores, which were derived from participants' responses on a four-point Likert scale. The GHQ-28 has high sensitivity and specificity for the identification of clinical diagnostic cases. ${ }^{17}$ The instrument has been widely used in disaster research, ${ }^{18,19}$ and it has been tested and validated in several cultural contexts, including Norwegian populations. ${ }^{20,21}$

\section{Personality, self-efficacy and social support}

Participants also completed the 44-item Big Five Inventory ${ }^{22}$ to measure personality dimensions, the General Self-Efficacy Scale ${ }^{23}$ to measure ability to cope with novel or demanding situations, and the Crisis Support Scale ${ }^{24}$ to assess support for the trauma. Negative social responses as well as support satisfaction were analysed separately from the other four items of the Crisis Support Scale, which were categorised as positive social support. ${ }^{25}$

\section{Other life events}

Participants completed a 12-item life-event inventory at the 24-month assessment. ${ }^{26}$ The number of negative life events that a participant experienced during the previous 12 months was recorded. Also, an additional item was included: whether participants witnessed or experienced a potentially traumatising event such as severe accident, armed robbery, physical violence, rape, war or another disaster in the past 12 months.

\section{Analysis of data}

We used chi-square to compare included and excluded individuals with regard to gender, age (over/under 44 years) and levels of education, employment, family constellations and pre-disaster contact with health professionals. The McNemar test for paired proportions was used to study the change in recalled threat intensity from 6 to 24 months. The prevalence of increasing intensity reports of death at the second assessment in different subgroups was compared using logistic regression. Multiple logistic regression analysis was used to study adjusted effects of several variables. Logistic regression analysis was used for significance testing of interactions. When studying differences in IES-R symptoms, a paired $t$-test was used to estimate changes within groups, whereas a difference between groups was tested with an independent sample $t$-test. The McNemar test for paired proportions was performed to examine the change in the proportion of participants with IES-R score $\geqslant 33$. All tests were two-tailed and differences were considered significant if $P<0.05$. The statistical analysis was performed with the software package SPSS version 14.0 for Windows. 
Table 2 Recalled threat intensity of the tsunami at 6 months and 24 months post-disaster $(n=416)$ based on the question 'How great do you think the danger was that you would die?'a

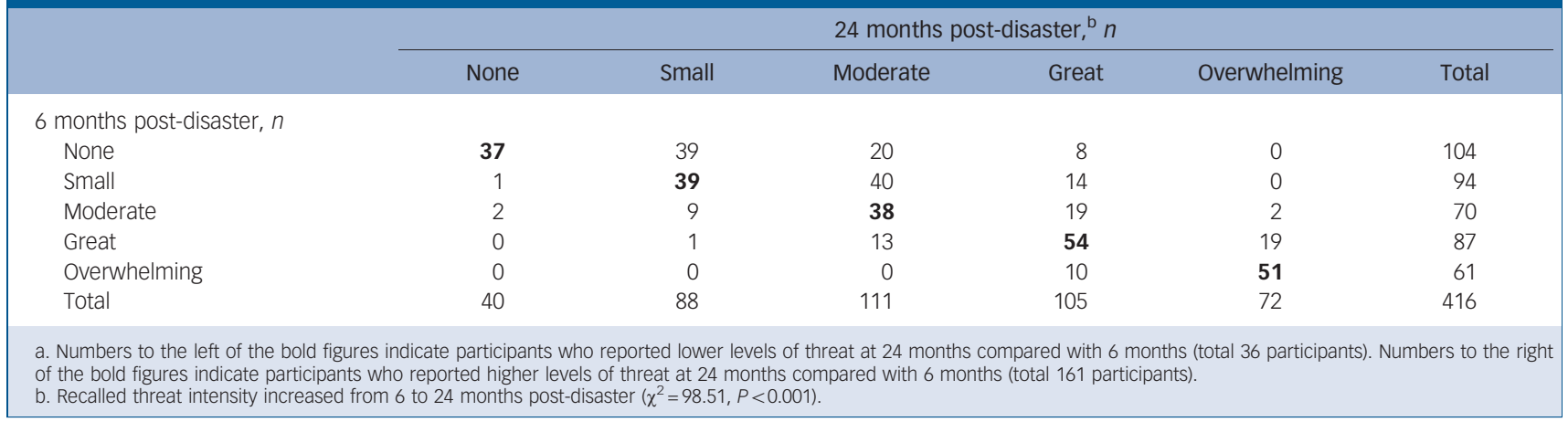

\section{Results}

Table 2 shows a cross-tabulation of responses after 6 months and 2 years to the question 'How great do you think the danger was that you would die?' Recalled threat intensity increased from 6 to 24 months post-disaster $\left(\chi^{2}=98.51, P<0.001\right)$.

Excluding the 61 respondents who endorsed 'overwhelming danger' during the first assessment, there were 355 participants who potentially could have changed their answer category to a higher degree of perceived danger from the first to the second assessment. Among these, $161(45.4 \%)$ declared a higher degree of danger at the second assessment.

Inversely, excluding the 104 respondents who endorsed 'no danger' during the first assessment, 312 participants could have potentially changed their answer category to a lower degree of perceived danger from the first to the second time of reporting. Among these, 36 or $11.5 \%$ endorsed a lower degree of perceived danger at the second assessment.

Increased recalled threat intensity was not significantly associated with age, gender, marital status, education level, exposure during the tsunami (i.e. caught or touched by waves; witnessing dead bodies; survivors with body injuries; people who searched for loved one's, abandoned children; uncertain of close relations' fate; loss of close relations) or immediate stress response (Criterion A2). Also, increased recalled threat intensity was not significantly associated with Big Five Inventory personality dimensions, self-efficacy, social support, 6- and 24-month GHQ-28 scores (total, somatisation, anxiety, social dysfunction, depression) or 6- and 24-month IES-R scores (total, intrusion, avoidance, hyperarousal). Nor was recalled threat intensity associated with other negative life events or potentially traumatising events that were experienced during the course of the study.

The only variable that was significantly associated with an increase in recalled threat intensity was changes in IES-R total symptom scores (Wald $=4.93, P=0.025$ ). A lack of improvement in PTSD symptoms was linked to an amplification of recalled perceived life threat at the second assessment.
This association was found to be robust for adjustments by other variables including age, gender, exposure to the tsunami, immediate stress response, exposure to other life events or potentially traumatising events, personality dimensions, general psychological distress, depressive symptoms, and 6-month IES-R total symptom scores, as well as each of the IES-R subscales. No significant interactions between changes in IES-R total symptom scores and other variables were found. Estimated changes in IES-R total symptom scores from 6 months to 2 years verified that participants who did not report increased threat intensity significantly improved in symptom severity, whereas those with amplification of recalled threat did not (Table 3).

Among those who did not report increased threat intensity, the proportion of participants with high symptom scores (IES-R $\geqslant 33$ ) decreased from $36.3 \%$ at 6 months to $26.8 \%$ at 24 months $\left(26.2 \%\right.$ decrease, $\left.\chi^{2}=9.53, P=0.002\right)$. Among those with amplification of recalled threat, the corresponding proportion increased from $33.1 \%$ at 6 months to $34.4 \%$ at 24 months (3.9\% increase, $\left.\chi^{2}=0.11, P=0.74\right)$.

\section{Discussion}

This study tested whether recalled threat intensity changes over time during the aftermath of a large disaster in a novel community sample that was exposed to a single, well-defined stressor. Compared with a baseline 6-month assessment, we found a greater intensity of recalled perceived life threat at a 2-year follow-up, which suggests that the memories for stressful events are amplified as time passes. Our findings support other notions about the inaccuracy of recall for stressful events. ${ }^{10,27}$

\section{Recall amplification process}

To understand why the recall of threat intensity increased over time in our sample, we examined the possible influence of disaster-related variables such as magnitude of exposure, immediate stress response and PTSD symptoms, as well as the

Table 3 Changes in Impact of Event Scale - Revised (IES-R) total symptom scores from 6 to 24 months in participants who did not report increased recalled threat intensity (no amplification) and those who did (amplification)

\begin{tabular}{|c|c|c|c|c|}
\hline & \multirow{2}{*}{$\begin{array}{l}\text { IES-R total at } 6 \text { months } \\
\text { Mean (s.d.) }\end{array}$} & \multirow{2}{*}{$\begin{array}{l}\text { IES-R total at } 24 \text { months } \\
\text { Mean (s.d.) }\end{array}$} & \multicolumn{2}{|c|}{ Change in IES-R } \\
\hline & & & Mean (s.d.) & Statistical test \\
\hline No amplification, $n=190$ & 25.95 (17.95) & $22.86(17.14)$ & $-3.08(12.53)$ & $t=3.4, P=0.001$ \\
\hline Amplification, $n=160$ & $24.91(17.04)$ & $24.90(16.91)$ & $-0.01(12.90)$ & $t=0.0, P=1.00$ \\
\hline Statistical test & $t=0.56, P=0.58$ & $t=-1.11, P=0.26$ & $t=-2.26, P=0.025$ & \\
\hline
\end{tabular}


possible influence of intra-individual variables such as age, gender, socioeconomic status, depression and other psychopathology, personality dimensions, self-efficacy and social support.

Results show that specific reports of exposure, such as being caught by waves or witnessing grotesque occurrences, did not significantly change reports of recalled threat over time. Individuals at the lower end of the threat intensity scale had the same risk for recall amplification as those in the middle or upper end. Contrary to other study findings, ${ }^{8,10}$ endorsements of DSM-IV A2 criteria, which involve having an immediate response of intense fear, helplessness or horror, did not influence changes in reported threats of death. Thus, even with the broad spectrum of exposure experiences in our sample, recall amplification seems to be virtually independent of exposure magnitude and type as well as the acuity of immediate stress response.

Amplification of recall was not related to PTSD symptom severity regardless if measured at 6 or 24 months post-disaster. Results held when we controlled for each PTSD symptom cluster separately, including the intrusion symptom dimension, which offers a potential construct overlap. Whereas other research found that individuals with higher scores of post-traumatic symptomatology have increased reports of stressful memories over time, ${ }^{5,7}$ our results did not.

Importantly, we found that recalled threat was differentially related to PTSD symptom reduction. Individuals with recall amplification did not improve in PTSD symptom severity from 6 to 24 months, whereas individuals who did not have recall amplification showed a reduction in PTSD symptoms over time.

The reduction in PTSD symptom level severity was rather small, even for the group of individuals that did not have recall amplification. However, the potential for improvement among those with few symptoms was low, which statistically capped the mean symptom reduction. With respect to the reduction in the number of individuals with high symptom scores, the difference between those without recall amplification (36.3\% to $26.8 \%$ ) and those who recalled higher life threat at the second assessment (no reduction) suggests clinical ramifications.

There are several possible theoretical explanations for this finding, most of which involve a bidirectional relationship between PTSD symptoms and memory process. For example, a lack of PTSD symptom improvement may drive recall amplification. ${ }^{28}$ Some participants may have amplified memories to justify their lack of improvement. Inversely, recall amplification may stifle PTSD symptom improvement. As participants' memories of the tsunami grew more disturbing, a parallel belief process may have emerged regarding prognosis.

Also, it is possible that recall amplification resulted from distorted cognitive processing because of the presence of other psychopathology. However, dimensions of psychological functioning such as anxiety, depression, and personality traits (e.g. neuroticism) were not related to changes in recalled threat. This is especially noteworthy with depression, where recall emphasises negative events. ${ }^{29}$ Furthermore, we tested for specific cognitive confounds with single items of the IES-R, such as 'I had trouble concentrating' and 'I tried to remove it from my memory', that yielded no significant effects. Nor did two items that measured sleep (trouble falling asleep, trouble staying asleep), which were specifically tested because of evidence that sleep effects memory consolidation. ${ }^{30}$ Thus, our results suggest that a lack of PTSD symptom improvement exclusively is associated with recall amplification.

Even though we controlled for a plethora of potential confounds, it is possible that recall amplification was influenced by an additional unmeasured construct, such as general autobiographical recall disturbance, which has been linked to extreme stress exposure. ${ }^{31,32}$ Also, recall amplification may occur for important events in general, as described by Frueh et $a l^{9}$ with regard to fishing stories: 'The tale often seems to grow taller on down the line'. Threat may be exaggerated over time as a result of exposure to media accounts, multiple retellings of the tsunami experience or other factors. But without objective evidence of actual threat, it is impossible to know which (if any) of a participant's self-report represents a 'true' occurrence of events.

\section{Methodological considerations}

This study has a number of methodological benefits. Virtually the entire population of Norwegians who were on Christmas holiday in South-East Asia during the 2004 tsunami was asked to participate in our study, reducing sample selection bias. Also, our community sample is probably not confounded by high rates of pre-existing psychopathology or other risk factors for PTSD. In fact, our sample had higher than average levels of socioeconomic status and education, which are known protective factors for psychopathology. Unlike other studies, our sample was exposed to a single, distinct event, and then participants were quickly removed from additional tsunami trauma experiences to their unaffected home communities in Norway. Thus, the impact of trauma is limited to the primary tsunami experience, not secondary stressors such as loss of home or livelihood. Finally, our sample has fewer reimbursement motives than military veterans. ${ }^{9}$ In fact, regardless of impairment level, our sample received affordable and easily accessible medical and psychiatric care from the Norwegian government as well as ample community support. $^{33}$

Limitations of our study include relatively low response and reply rates, which imply that our results must be interpreted with caution. However, lack of participation was mostly because of perceived study irrelevance to personal situations such as no exposure to the tsunami wave. Also, disaster exposures as well as immediate stress response data were ascertained retrospectively 6 months post-disaster, and we only used self-report measures. Our results may not be applicable to other populations that were exposed to chronic stressors. Furthermore, our assessment of perceived threat intensity with a single item lacks multi-method, multi-informant reliability and validity estimates. Also, we did not address important mechanisms of recall distortion such as appraisal errors. ${ }^{34}$

\section{Implications}

Our study has several important implications. Assessment of a Criterion A stressor is principally based on an individual's retrospective self-report without collateral information of the stressful experience. Our findings suggest that such self-reports may be imprecise and unstable, which questions the diagnostic validity of PTSD. Indeed, it is possible for an individual to receive a PTSD diagnosis without having been exposed to a Criterion A stressor because some individuals may report an event as more severe than if they had been questioned at an earlier point in time. Subsequent treatment planning, healthcare rights, disability payments, classification of participants into research groups, among other issues, are affected by such erroneous diagnosis.

Spitzer et $a l^{35}$ have argued for a revised PTSD criteria set for the DSM-V. Proposed changes include the requirement of symptom development within 1 week of a traumatic event or, in the case of delayed onset, symptom debut associated with a thematically related trauma. Although this revision may help with linking symptoms to specific stressful experiences (i.e. diagnostic 
specificity) it will not correct for the inaccuracy of retrospective accounts of life threat.

Cognitive-behavioural therapy is recommended for PTSD treatment. ${ }^{36}$ Our findings suggest that cognitive approximations that promote objective threat evaluation would be helpful for such trauma treatment. An active reconstruction of the factual course of events may be a useful tool in this process. ${ }^{37}$

In conclusion, our findings suggest that recall amplification of perceived life threat occurs in the general population when exposed to a distinct stressful event. This phenomenon raises critical questions about the diagnostic validity of PTSD as currently defined by the ICD-10 and the DSM-IV. Furthermore, recall amplification of perceived life threat appears to interrelate with the PTSD healing process in important, possibly harmful ways. Additional stressful event recall research should widen the time horizon and frequency of assessments, and examine the influence of other types of stress exposure as well as moderators and mediators of recall amplification.

\section{Funding}

This study was supported by the Norwegian Directorate of Health and Social Affairs.

\section{Acknowledgements}

The authors thank the many staff members at the centre for their contribution to the preparation of the study and Ajmal Hussain for his support in conducting the study.

Trond Heir, MD, PhD, Auran Piatigorsky, PhD, Lars Weisæth, MD, PhD, Norwegian Centre for Violence and Traumatic Stress Studies, University of Oslo, Norway

Correspondence: Trond Heir, Norwegian Centre for Violence and Traumatic Stress Studies, Building 48, Kirkeveien 166, N-0407 Oslo, Norway. Email: trond.heir@medisin.uio.no

First received 27 June 2008, final revision 6 Nov 2008, accepted 17 Dec 2008

\section{References}

1 World Health Organization. The ICD-10 Classification of Mental and Behavioural Disorders: Clinical Descriptions and Diagnostic Guidelines. WHO, 1992.

2 American Psychiatric Association. Diagnostic and Statistical Manual of Mental Disorder (4th edn) (DSM-IV). APA, 1994

3 van der Kolk BA. Trauma and memory. In Traumatic Stress: The Effects of Overwhelming Experience on Mind, Body and Society (eds BA Van der Kolk AC McFarlane \& L Weisaeth): 279-302. Guilford Press, 1996.

4 Southwick SM, Morgan CA, Nicolaou AL, Charney DS. Consistency of memory for combat-related traumatic events in veterans of Operation Desert Storm. Am J Psychiatry 1997; 154: 173-7.

5 Roemer L, Litz BT, Orsillo SM, Ehlich PJ, Friedman MJ. Increases in retrospective accounts of war-zone exposure over time: the role of PTSD symptom severity. J Trauma Stress 1998; 11: 597-605.

6 Wessely S, Unwin C, Hotopf M, Hull L, Ismail K, Nicolaou V, et al. Stability of recall of military hazards over time. Evidence from the Persian Gulf War of 1991. Br J Psychiatry 2003; 183: 314-22.

7 Engelhard IM, van den Hout MA, McNally RJ. Memory consistency for traumatic events in Dutch soldiers deployed to Iraq. Memory 2008; 16: 3-9.

8 Krinsley KE, Gallagher JG, Weathers FW, Kutter CJ, Kaloupek DG. Consistency of retrospective reporting about exposure to traumatic events. J Trauma Stress 2003; 16: 399-409.

9 Frueh $\mathrm{BC}$, Hamner MB, Cahill SP, Gold PB. Apparent symptom over-reporting in combat veterans evaluated for PTSD. Clin Psychol Rev 2000; 20: 853-85.

10 Hepp U, Gamma A, Milos G, Eich D, Ajdacic-Gross V, Rössler W, et al. Inconsistency in reporting potentially traumatic events. Br J Psychiatry 2006; 188: $278-83$.
11 Ouimette P, Read J, Brown PJ. Consistency of retrospective reports of DSM-IV Criterion A traumatic stressors among substance use disorder patients. J Trauma Stress 2005; 18: 43-51.

12 Porter S, Peace KA. The scars of memory. A prospective, longitudinal investigation of the consistency of traumatic and positive emotional memories in adulthood. Psychol Sci 2007; 18: 435-41.

13 Heir T, weisaeth L. Acute disaster exposure and mental health complaints of Norwegian tsunami survivors 6 months post disaster. Psychiatry 2008; 71: 266-76

14 Portney LG, Watkins MP. Foundations of Clinical Research: Applications to Practice (2nd edn). Prentice Hall Health, 2000.

15 Weiss DS. The Impact of Event Scale - Revised. In Assessing Psychological Trauma and PTSD (eds JP Wilson \& TM Keane): 168-89. Guilford Press, 2004

16 Creamer M, Bell R, Failla S. Psychometric properties of the Impact of Event Scale - Revised. Behav Res Ther 2003; 41: 1489-96.

17 Goldberg DP, Hillier VF. A scaled version of the General Health Questionnaire. Psychol Med 1979; 9: 139-45.

18 Hull AM, Alexander DA, Klein S. Survivors of the Piper Alfa oil platform disaster: long-term follow-up study. Br J Psychiatry 2002; 181: 433-8.

$19 \mathrm{Cao} \mathrm{H}, \mathrm{McF}$ arlane AC, Klimidis S. Prevalence of psychiatric disorder following the 1988 Yun Nan (China) earthquake: the first 5-month period. Soc Psychiatry Psychiatr Epidemiol 2003; 38: 204-12.

20 Thiis-Evensen E, Wilhelmsen I, Hoff GS, Blomhoff S, Sauar J. The psychological effect of attending a screening program for colorectal polyps. Scand J Gastroenterol 1999; 34: 103-9.

21 Skari H, Skreden M, Malt UF, Dalholt M, Ostensen AB, Egeland T, et al. Comparative levels of psychological distress, stress symptoms, depression and anxiety after childbirth: a prospective population-based study of mothers and fathers. BJOG 2002; 109: 1154-63.

22 John OP, Donahue EM. The Big Five Inventory: Construction and Validation. Institute of Personality and Social Research, 1998.

23 Schwarzer R, Jerusalem M. Generalized self-efficacy scale. In Measures in Health Psychology: A User's Portfolio. Causal and Control Beliefs (eds J Weinman, S Wright \& M Johnston): 35-7. nfer-Nelson, 1995.

24 Joseph S, Andrews B, Williams R, Yule W. Crisis support and psychiatric symptomatology in adult survivors of the Jupiter cruise ship disaster. Br J Clin Psychol 1992; 31: 63-73.

25 Andrews B, Brewin CR, Rose S. Gender, social support, and PTSD in victims of violent crime. J Trauma Stress 2003; 16: 421-7.

26 Brugha $T$, Bebbington $\mathrm{P}$, Tennant $\mathrm{C}$, Hurry J. The list of threatening experiences: a subset of 12 life events categories with considerable long term contextual threat. Psychol Med 1985; 15: 189-94.

27 McHugh PR, Treisman G. PTSD: a problematic diagnostic category. J Anxiety Disord 2007; 21: 211-22.

28 King DW, King LA, Erickson DJ, Huang MT, Sharkansky EJ, Wolfe J. Posttraumatic stress disorder and retrospectively reported stressor exposure: a longitudinal prediction model. J Abnorm Psychol 2000; 109: 624-33.

29 Colombel F. [Memory bias and depression: a critical commentary]. Encephale 2007; 33: 242-8.

30 Stickgold R, Walker MP. Sleep and memory: the ongoing debate. Sleep 2005; 28: 1225-7.

31 Moore SA, Zoellner LA. Overgeneral autobiographical memory and traumatic events: an evaluative review. Psychol Bull 2007; 133: 419-37.

32 Kleim B, Ehlers A. Reduced autobiographical memory specificity predicts depression and posttraumatic stress disorder after recent trauma. J Consult Clin Psychol 2008; 76: 231-42.

33 Hjemdal OK. The Regular Practitioners Follow-up of the Tsunami Exposed Individuals (Report no. 1/2007). [Report in Norwegian, English summary.] Norwegian Centre for Violence and Traumatic Stress Studies, 2007.

34 O’Donnell ML, Elliott P, Wolfgang BJ, Creamer M. Posttraumatic appraisals in the development and persistence of posttraumatic stress symptoms. J Traumatic Stress 2007; 20: 173-82.

35 Spitzer RL, First MB, Wakefield JC. Saving PTSD from itself in DSM-V. J Anxiety Disord 2007; 21: 233-41.

36 Bradley R, Greene J, Russ E, Dutra L, Westen D. A multidimensional metaanalysis of psychotherapy for PTSD. Am J Psychiatry 2005; 162: 214-27.

37 Heir T, Weisaeth L. Back to where it happened: self-reported symptom improvement of tsunami survivors who returned to the disaster area. Prehospital Disaster Med 2006; 21: 59-63. 